\title{
Suitability of Primary Anastomosis for Colorectal Perforation
}

\author{
Hiroshi Asano $^{1}$ (D) $\cdot$ Hiroyuki Fukano ${ }^{1} \cdot$ Yasuhiro Ohara $^{1} \cdot$ Nozomi Shinozuka $^{1}$
}

Accepted: 22 November 2018 / Published online: 3 December 2018

(C) The Author(s) 2018

\begin{abstract}
Colorectal perforation is often treated with colostomy without primary anastomosis due to the risk of anastomotic leakage. However, colostomy affects the patients' quality of life and may cause complications during closure. This study aimed to examine the suitability of primary anastomosis for colorectal perforation and determine the risk factors for postoperative anastomotic leakage. We retrospectively examined patients who underwent emergency surgery for colorectal perforation associated with generalized peritonitis between April 2007 and March 2017. Cases with iatrogenic or traumatic causes were excluded. Patients were divided into primary anastomosis and colostomy groups. Their age, time after onset, preoperative sequential organ failure assessment (SOFA) score, perforation site, cause of perforation, shock, intraperitoneal infection, steroid use, chronic renal failure, and mortality were compared. Subgroup analysis of the primary anastomosis group was performed to identify risk factors for anastomotic leakage. The cohort comprised 232 patients (112 men, 120 women; mean age, 73 years). Primary anastomosis and colostomy were performed in 27 and 205 patients, respectively. The primary anastomosis group consisted mainly of men with right colonic perforation. Of the 27 patients who underwent primary anastomosis, anastomotic leakage occurred in 5 (18.5\%). Patients with anastomotic leakage demonstrated high SOFA scores $(4.2$ vs. $1.9, p<0.05)$ and Hinchey stage IV disease $(80 \%$ vs. $27.3 \%, p<0.05)$. Of the 5 patients with anastomotic leakage, 2 died. Anastomotic leakage after primary anastomosis for colorectal perforation occurs in patients with septic complications or severe intraperitoneal infection. Therefore, primary anastomosis should be avoided in this patient population.
\end{abstract}

Keywords Colorectal perforation $\cdot$ Primary anastomosis $\cdot$ SOFA $\cdot$ Anastomotic leakage

\section{Background}

Colorectal perforation is a frequent fatal condition [1-4] that has been extensively studied in terms of background factors, such as age and underlying disease [1, 4]; intraoperative findings, such as intraperitoneal infection [2]; and prognostic factors, such as the sequential organ failure assessment (SOFA)

This article is part of the Topical Collection on Surgery

Hiroshi Asano

hiroshia@saitama-med.ac.jp

Hiroyuki Fukano

hirof53530@yahoo.co.jp

Yasuhiro Ohara

y_ohara@saitama-med.ac.jp

Nozomi Shinozuka

zukasino@saitama-med.ac.jp

1 Department of General Surgery, Saitama Medical University, 38 Morohongou, Moroyama, Irumagun, Saitama 350-0495, Japan score. Treatment of colorectal perforation consists of intraperitoneal lavage and repair of the perforation site. In addition, given the risk of anastomotic leakage, colostomy is selectively performed in many cases. However, in patients who are beyond the acute phase, colostomy delays their reintegration into society, affects their daily activities, requires reoperation after closure, and poses numerous other problems. In light of these problems, primary anastomosis is sometimes performed without colostomy $[5,6]$. However, the suitability of primary anastomosis remains controversial. Although several previous case reports have demonstrated that primary anastomosis can be performed without complications in patients with no risk factors, such as shock or immunosuppression $[5,6]$, none have examined patients that do develop anastomotic leakage. While we have comprehensively assessed primary anastomosis based on factors such as perforation site, general condition, and degree of infection, consistent standards have not yet been established. Furthermore, surgeons have differing opinions on the suitability of primary anastomosis, even when examining the same patient. Consequently, the standardization of operative procedures is far from a reality. Therefore, the present 
study retrospectively examined cases of primary anastomosis for colorectal perforation to determine risk factors for anastomotic leakage and the suitability of primary anastomosis.

\section{Methods}

We retrospectively examined patients who underwent emergency surgery for colorectal perforation with generalized peritonitis at the Department of General Surgery, Saitama Medical University, Moroyama, Japan, between April 2007 and March 2017. This study was approved by the Institutional Review Board of Saitama Medical University Hospital (No. 18031). Cases with iatrogenic or traumatic causes were excluded. Patients who underwent primary anastomosis without colostomy were defined as the primary anastomosis group, while patients who underwent any form of colostomy were defined as the colostomy group. These two groups were compared in terms of age, time after onset, preoperative SOFA score, perforation site, cause of perforation, shock, intraperitoneal infection, steroid use, chronic renal failure, and mortality. We also performed the same comparisons within the primary anastomosis group between patients with and without anastomotic leakage to determine possible risk factors. Intraperitoneal infection was assessed in accordance with the Hinchey classification [7], with Hinchey III defined as generalized peritonitis associated with purulent ascites and Hinchey IV defined as generalized peritonitis associated with fecal matter. Shock was defined as a mean blood pressure $\leq 65 \mathrm{mmHg}$ despite massive transfusion and catecholamine administration upon entering the operating room. In cases of reoperation, anastomotic dehiscence was diagnosed based on intraoperative findings. In cases of primary operation, anastomotic leakage was diagnosed based on computed tomography findings of increased free air and abscess formation surrounding the anastomosis site.

\section{Surgical Technique}

In all cases, surgery for colorectal perforation was performed via laparotomy. The intestine was anastomosed with an autosuture device or an automatic anastomotic device. In principle, colostomy consisted of the Hartmann's procedure, in which the perforation site is resected and an end colostomy is formed from the proximal end. However, in some cases, the intestine was anastomosed after resection of the perforation site, and the stoma was formed at the proximal diversion. In other cases, the perforation site was directly taken out of the body to make a colostomy (exteriorization). The peritoneum was washed with $\geq 10,000 \mathrm{ml}$ of saline solution, and a closed drain was implanted in the pelvic floor. To close the abdomen, the muscle layer was closed with interrupted monofilament absorbable sutures, and the skin was closed with subcutaneous monofilament absorbable sutures after washing the subcutaneous tissue with $1000 \mathrm{ml}$ of saline solution. Carbapenem antibiotics were administered prior to surgery.

\section{Statistical Analysis}

Statistical analysis was performed using Excel (Microsoft, Seattle, WA) and BellCurve for Excel (Social Survey Research Information Co., Ltd., Tokyo, Japan). Continuous variables and categorical variables were examined using the Mann-Whitney $U$ test and the $\chi^{2}$ test, respectively. Statistical significance was defined as $p<0.05$.

\section{Results}

A total of 232 patients underwent emergency surgery for colorectal perforation with generalized peritonitis during the study period. The cohort comprised of 112 men and 120 women with a mean age of 73.0 years. The most common cause of perforation was diverticulum (79 patients), followed by malignancy (72 patients) and idiopathic cause (70 patients). The most common perforation sites were the sigmoid colon (138 patients) and the rectum (45 patients), and $88 \%$ of cases occurred in the left colon (Table 1). Primary anastomosis and colostomy were performed in 27 and 205 patients, respectively. Colostomy was performed with Hartmann's procedure, exteriorization, and proximal diversion in 173,17 , and 15 patients, respectively. Compared to the colostomy group, the primary anastomosis group had a significantly higher percentage of men and perforation in the right colon. Shock occurred in a total of 45 patients, almost entirely among patients who underwent colostomy. In the primary anastomosis group, shock occurred in only 1 patient (Table 2). A

Table 1 Characteristics

of patients with

\begin{tabular}{ll}
\hline No. of patients & 232 \\
Age (years) & 73.0 \\
Sex (M/F) & $112 / 120$ \\
In-hospital mortality (\%) & $37(16 \%)$ \\
Cause of perforation & \\
$\quad$ Diverticulum & 79 \\
Malignancy & 72 \\
Idiopathic & 70 \\
Ulcer & 5 \\
Ischemia & 3 \\
Others & 3 \\
Site of perforation & \\
Rectum & 45 \\
Sigmoid & 138 \\
Descending & 20 \\
Transverse & 12 \\
Ascending & 14 \\
Cecum & 3 \\
\hline
\end{tabular}


Table 2 Comparisons between the anastomosis and colostomy groups

\begin{tabular}{llll}
\hline & Anastomosis & Colostomy & $p$ value \\
\hline No. of patients & $n=27$ & $n=205$ & \\
Age (years) & 72.8 & 73.0 & 0.981 \\
Sex (M:F) & $21: 6$ & $91: 114$ & 0.002 \\
Time to surgery since onset (h) & 17.3 & 23.6 & 0.460 \\
SOFA score & 3.0 & 2.3 & 0.321 \\
Left colon & $13(48 \%)$ & $190(92 \%)$ & 0.001 \\
Malignancy & $12(44 \%)$ & $60(29 \%)$ & 0.109 \\
Shock & $1(4 \%)$ & $44(21 \%)$ & 0.028 \\
Hinchey IV & $10(37 \%)$ & $84(41 \%)$ & 0.695 \\
Steroid use & $1(4 \%)$ & $21(10 \%)$ & 0.275 \\
Chronic renal failure & $3(11 \%)$ & $25(12 \%)$ & 0.871 \\
In-hospital mortality & $5(19 \%)$ & $32(16 \%)$ & 0.698 \\
\hline
\end{tabular}

SOFA, sequential organ failure assessment

total of 37 patients (16\%) died in the hospital, but in-hospital mortality did not differ between the primary anastomosis (19\%) and colostomy groups (16\%). Anastomotic leakage occurred in 5 of 27 primary anastomosis patients $(18.5 \%)$. Compared to patients without anastomotic dehiscence, patients with anastomotic dehiscence more commonly demonstrated high SOFA scores $(4.2$ vs. $1.9, p<0.059)$ and Hinchey IV disease $(80 \%$ vs. $27.3 \%, p<0.05$ ) (Table 3 ). Table 4 shows the details of the 5 patients with anastomotic leakage. Of these 5 patients, 4 had SOFA scores of $\geq 3$, and 2 died.

\section{Discussion}

Anastomotic leakage is a serious and frequently fatal complication [8] that occurs in 2.4-12\% of standard colorectal surgery cases [9-12]. However, in patients with colorectal perforation, it is significantly more frequent [13]. Anastomotic leakage is

Table 3 Potential risk factors for anastomotic leakage

\begin{tabular}{llll}
\hline & Leakage & No leakage & $p$ value \\
\hline No. of patients & 5 & 22 & \\
Age (years) & 67.4 & 74.0 & 0.274 \\
Time to surgery after onset (h) & 10 & 18 & 0.130 \\
SOFA score & 4.2 & 1.9 & 0.039 \\
Left colon & $2(40 \%)$ & $11(50 \%)$ & 0.686 \\
Malignancy & $4(80 \%)$ & $8(36 \%)$ & 0.076 \\
Shock & $0(0 \%)$ & $1(5 \%)$ & 0.627 \\
Hinchey IV & $4(80 \%)$ & $6(27 \%)$ & 0.028 \\
Steroid use & $0(0 \%)$ & $1(5 \%)$ & 0.627 \\
Chronic renal failure & $1(20 \%)$ & $2(9 \%)$ & 0.484 \\
In-hospital mortality & $2(40 \%)$ & $3(14 \%)$ & 0.171 \\
\hline
\end{tabular}

SOFA, sequential organ failure assessment typically avoided by performing a colostomy followed by a secondary operation to close it. However, for patients who are past the acute phase, colostomy poses many disadvantages, such as negative effects on daily activities and delayed reintegration into society. When performing closure, the operative procedure must account for complications such as anastomotic leakage. In comparisons between primary anastomosis and colostomy with secondary anastomosis for perforated diverticulitis, Salem et al. [14] reported that complications were less frequent after primary anastomosis than secondary anastomosis. However, primary anastomosis has a poor reputation due to a high frequency of anastomotic leakage, up to $28 \%$ [13]. In addition, primary anastomosis is not definitely suitable for all patients. Therefore, strict indications must be established to ensure that primary anastomosis is not performed in patients with a high risk of anastomotic leakage. In the present study, anastomotic leakage occurred in 5 of 27 patients (18.5\%) who underwent primary anastomosis. These patients demonstrated significantly higher preoperative SOFA scores and more frequently had Hinchey IV disease, suggesting that these may be key factors to consider before selecting patients for primary anastomosis.

A number of previous studies have discussed the suitability of primary anastomosis for colorectal perforation. Biondo et al. [5] believe that primary anastomosis should not be performed for patients with cirrhosis, chronic kidney disease, or immunosuppression. They reported that of 33 Hinchey III-IV patients without these comorbidities who underwent primary anastomosis, leakage occurred in only 1 patient. Vascular lesions, immunosuppression, and recent laparotomy were identified as risk factors for leakage after primary anastomosis in a study by Richter et al. [6], where only 1 of 34 patients (2.9\%) without these risk factors experienced anastomotic leakage. In their study, the mortality in patients undergoing primary anastomosis was $11 \%$, and the authors concluded that anastomotic leakage did not affect prognosis. However, no study has specifically examined patients in whom anastomotic leakage occurred. In the present study, the clinical backgrounds of the patients with anastomotic leakage suggested that preoperative SOFA score and Hinchey IV disease may be risk factors. Factors involved in post-colectomy anastomotic leakage include not only intraoperative complications, such as incomplete anastomosis, anastomotic blood supply, and excessive tension and bleeding in the anastomosis site, but also patient factors such as chronic diseases (diabetes, cirrhosis, renal failure, etc.), steroid use, and sex $[15,16]$. Among these factors, anastomotic blood supply seems to play a particularly significant role. In a typical colectomy, anastomotic leakage occurs because of intraoperative technical lapses, such as compromise of anastomotic blood supply due to the ligation of mesenteric vessels. However, in colorectal perforation, anastomotic leakage is considered to involve microangiopathy associated with sepsis. Sepsis causes vascular endothelial dysfunction and results in microangiopathy associated with hypercoagulation [17]. These complications become even more serious if sepsis 
Table 4 Details of patients with anastomotic leakage

\begin{tabular}{lllllll}
\hline $\begin{array}{l}\text { Age/ } \\
\text { sex }\end{array}$ & Cause of perforation & Location & Surgical procedure & Hinchey & SOFA & Outcome \\
\hline $67, \mathrm{M}$ & Malignancy & Ascending & Ileocecal resection & III & 7 & Death \\
$88, \mathrm{M}$ & Malignancy & Sigmoid & Sigmoidectomy & IV & 5 & Death \\
$62, \mathrm{M}$ & Malignancy & Ascending & Right hemicolectomy & IV & 5 & Alive \\
$49, \mathrm{M}$ & Malignancy & Ascending & Right hemicolectomy & IV & 3 & Alive \\
$71, \mathrm{M}$ & Diverticulum & Sigmoid & Sigmoidectomy & IV & 1 & Alive \\
\hline
\end{tabular}

SOFA, sequential organ failure assessment progresses into disseminated intravascular coagulation or septic shock. Therefore, primary anastomosis should only be performed in patients with a SOFA score less than 2 and without sepsis. Furthermore, even in patients who are in a stable condition prior to surgery, the effects of infection can be compounded by the open surgical field, thereby leading to organ dysfunction, more severe coagulation disorders, and subsequent postoperative shock. Although postoperative septic shock is difficult to predict, Hinchey IV disease and a SOFA score $\geq 3$ have been reported as risk factors [18]. Therefore, primary anastomosis should not be performed in patients with Hinchey IV disease, as it may lead to particularly severe cases of sepsis.

In this study, in 3 of the 5 patients with anastomotic leakage, the perforation site was in the right colon. Although guidelines for selecting the operative procedure in emergency colon surgery have not been established, emergency surgery for the right colon often consists of ileocolonic anastomosis [19, 20], while emergency surgery for the left colon typically involves a colostomy without anastomosis $[21,22]$. This difference in operative procedure selection seems to be rooted in the belief that anastomotic leakage in the right colon is uncommon. However, one study has found anastomotic leakage to be more common in emergency surgery for the right colon than for the left [23]. Therefore, anastomosis should not be routinely performed in the right colon. In the present study, although colostomy was selected for $50 \%$ of patients with perforation in the right colon, anastomotic leakage occurred in $20 \%$. However, the high rate of high SOFA scores among patients with anastomotic leakage suggests that risk assessment [24] can improve therapeutic outcomes without routinely performing primary anastomosis during right colon surgery.

The present study was limited in that it was not a randomized controlled trial, meaning that anastomosis was not performed for empirically high-risk patients. In particular, only 1 patient who underwent anastomosis presented with preoperative shock, which was therefore not necessarily demonstrated to be a true risk factor. In addition, the present study did not include enough patients with renal failure or those using steroids. More such patients must be enrolled and analyzed.

Finally, primary anastomosis should not be performed in patients with sepsis. Even if a patient does not have sepsis prior to surgery, they may develop postoperative sepsis in the event of pronounced infection, such as fecal peritonitis. Chronic disease (cirrhosis, renal failure, etc.) and steroid use, which have been previously reported as risk factors for anastomotic leakage in primary anastomosis, were not risk factors in the present study. However, anastomosis is also regarded with caution during typical colectomy in patients with cirrhosis or renal failure and those using steroids; therefore, primary anastomosis for colorectal perforation should be avoided in these patients [25]. Furthermore, anastomotic leakage is affected not only by blood supply, but also by other factors such as internal intestinal pressure. Based on this data, and the fact that anastomotic leakage also occurs to a noticeable extent in typical colectomy, comprehensive assessment of patients is warranted.

\section{Conclusion}

In this study, anastomotic leakage after primary anastomosis for colorectal perforation occurs in patients with septic complications or severe intraperitoneal infection. Therefore, primary anastomosis should be avoided in this patient population. Furthermore, since the mortality rate of patients with anastomotic leakage is high, cautious assessment of the primary anastomosis is necessary.

\section{Compliance with Ethical Standards}

Conflict of Interest The authors declare that they have no conflict of interest.

Ethical Approval All procedures performed in studies involving human participants were in accordance with the ethical standards of the institutional and/or national research committee and with the 1964 Helsinki Declaration and its later amendments or comparable ethical standards.

For this type of study formal consent is not required.

Consent for Publication Not applicable.

Open Access This article is distributed under the terms of the Creative Commons Attribution 4.0 International License (http:// creativecommons.org/licenses/by/4.0/), which permits unrestricted use, distribution, and reproduction in any medium, provided you give appropriate credit to the original author(s) and the source, provide a link to the Creative Commons license, and indicate if changes were made. 


\section{References}

1. Kriwanek S, Armbruster C, Beckerhinn P, Dittrich K. Prognostic factors for survival in colonic perforation. Int J Color Dis. 1994;9: 158-62. https://doi.org/10.1007/BF00290194.

2. Bielecki K, Kamiński P, Klukowski M. Large bowel perforation: morbidity and mortality. Tech Coloproctol. 2002;6:177-82. https:// doi.org/10.1007/s101510200039.

3. Biondo S, Parés D, Martí Ragué J, De Oca J, Toral D, Borobia FG, et al. Emergency operations for nondiverticular perforation of the left colon. Am J Surg. 2002;183:256-60. https://doi.org/10.1016/ S0002-9610(02)00780-8.

4. Yamamoto T, Kita R, Masui H, Kinoshita H, Sakamoto Y, Okada $\mathrm{K}$, et al. Prediction of mortality in patients with colorectal perforation based on routinely available parameters: a retrospective study. World J Emerg Surg. 2015;10:24.

5. Biondo S, Jaurrieta E, Martí Ragué J, Ramos E, Deiros M, Moreno $\mathrm{P}$, et al. Role of resection and primary anastomosis of the left colon in the presence of peritonitis. Br J Surg. 2000;87:1580-4. https:// doi.org/10.1046/j.1365-2168.2000.01556.x.

6. Richter S, Lindemann W, Kollmar O, Pistorius GA, Maurer CA, Schilling MK. One-stage sigmoid colon resection for perforated sigmoid diverticulitis (Hinchey stages III and IV). World J Surg. 2006;30:1027-32. https://doi.org/10.1007/s00268-005-0439-5.

7. Hinchey EJ, Schaal PG, Richards GK. Treatment of perforated diverticular disease of the colon. Adv Surg. 1978;12:85-109.

8. Choi H-KK, Law W-LL, Ho JWCC. Leakage after resection and intraperitoneal anastomosis for colorectal malignancy: analysis of risk factors. Dis Colon Rectum. 2006;49:1719-25.

9. Kanellos I, Blouhos K, Demetriades H, Pramateftakis MG, Mantzoros I, Zacharakis E, et al. The failed intraperitoneal colon anastomosis after colon resection. Tech Coloproctol. 2004;8:53-5.

10. Isbister WH. Anastomotic leak in colorectal surgery: a single surgeon's experience. ANZ J Surg. 2001;71:516-20. https://doi.org/ 10.1046/j.1440-1622.2001.02189.x.

11. Law WI, Chu KW, Ho JW, Chan CW. Risk factors for anastomotic leakage after low anterior resection with total mesorectal excision. Am J Surg. 2000;179:92-6. https://doi.org/10.1016/S00029610(00)00252-X.

12. Pakkastie TE, Luukkonen PE, Järvinen HJ. Anastomotic leakage after anterior resection of the rectum. Eur J Surg. 1994;160:293-7.

13. Zingg U, Pasternak I, Dietrich M, Seifert B, Oertli D, Metzger U. Primary anastomosis vs Hartmann's procedure in patients undergoing emergency left colectomy for perforated diverticulitis. Color Dis. 2010;12:54-60. https://doi.org/10.1111/j.1463-1318.2008.01694.x.

14. Salem L, Flum DR. Primary anastomosis or Hartmann's procedure for patients with diverticular peritonitis? A systematic review. Dis Colon Rectum. 2004;47:1953-64.

15. Nikolian VC, Kamdar NS, Regenbogen SE, Morris AM, Byrn JC, Suwanabol PA, et al. Anastomotic leak after colorectal resection: a population-based study of risk factors and hospital variation. Surg. 2017;161:1619-27. https://doi.org/10.1016/j.surg.2016.12.033.

16. Kingham TP, Pachter HL. Colonic anastomotic leak: risk factors, diagnosis, and treatment. J Am Coll Surg. 2009;208:269-78.

17. Okamoto K, Tamura T, Sawatsubashi Y. Sepsis and disseminated intravascular coagulation. J Intensive Care. 2016;4:1-8.

18. Asano H, Takayama T, Ogino N, Fukano H, Ohara Y, Shinozuka N. Study of postoperative shock predictors in colorectal perforation. J Japanese Coll Surg. 2016;41:891-6.

19. Lee YM, Law WL, Chu KW, Poon RTP. Emergency surgery for obstructing colorectal cancers: a comparison between right-sided and left-sided lesions. J Am Coll Surg. 2001;192:719-25.

20. Irvin TT, Greaney MG. The treatment of colonic cancer presenting with intestinal obstruction. Br J Surg. 1977;64:741-4. https://doi. org/10.1002/bjs.1800641015.

21. Deans GT, Krukowski ZH, Irwin ST. Malignant obstruction of the left colon. Br J Surg. 1994;81:1270-6. https://doi.org/10.1002/bjs. 1800810905.

22. Timmermans DR, Gooszen AW, Geelkerken RH, Tollenaar RA, Gooszen HG. Analysis of the variety in surgeons' decision strategies for the management of left colonic emergencies. Med Care. 1997;35:701-13. https://doi.org/10.1097/00005650199707000-00004.

23. Biondo S, Parés D, Frago R, Martí-Ragué J, Kreisler E, De Oca J, et al. Large bowel obstruction: predictive factors for postoperative mortality. Dis Colon Rectum. 2004;47:1889-97.

24. Biondo S, Parés D, Kreisler E, Ragué JM, Fraccalvieri D, Ruiz AG, et al. Anastomotic dehiscence after resection and primary anastomosis in left-sided colonic emergencies. Dis Colon Rectum. 2005;48:2272-80.

25. Eriksen TF, Lassen CB, Gögenur I. Treatment with corticosteroids and the risk of anastomotic leakage following lower gastrointestinal surgery: a literature survey. Color Dis. 2014;16:0154-60. https:// doi.org/10.1111/codi.12490. 